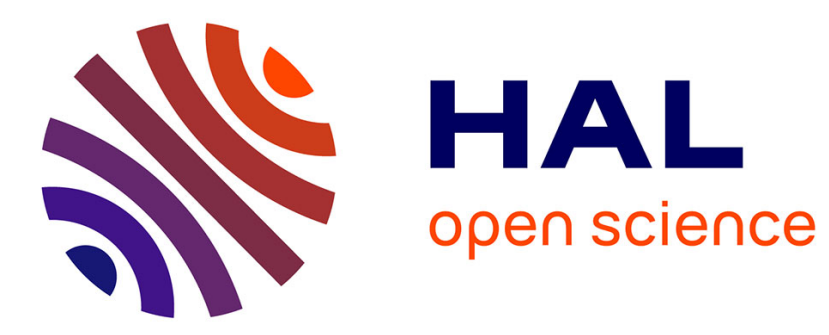

\title{
Optimisation de la mesure de figures de pôles en diffraction $\mathrm{X}$
}

\author{
F. Wagner, M. Stosse, C. Nauer-Gerhardt
}

\section{To cite this version:}

F. Wagner, M. Stosse, C. Nauer-Gerhardt. Optimisation de la mesure de figures de pôles en diffraction X. Journal de Physique IV Proceedings, 1996, 06 (C4), pp.C4-307-C4-317. 10.1051/jp4:1996429 . jpa-00254312

\section{HAL Id: jpa-00254312 https://hal.science/jpa-00254312}

Submitted on 1 Jan 1996

HAL is a multi-disciplinary open access archive for the deposit and dissemination of scientific research documents, whether they are published or not. The documents may come from teaching and research institutions in France or abroad, or from public or private research centers.
L'archive ouverte pluridisciplinaire HAL, est destinée au dépôt et à la diffusion de documents scientifiques de niveau recherche, publiés ou non, émanant des établissements d'enseignement et de recherche français ou étrangers, des laboratoires publics ou privés. 


\section{Optimisation de la mesure de figures de pôles en diffraction $\mathbf{X}$}

F. Wagner, M. Stosse et C. Nauer-Gerhardt*

CMS/ISGMP, Université de Metz, Ile du Saulcy, 57045 Metz cedex, France

* SIEMENS AG, AUT 37 PM, P.O. Box 21 1262, 76187 Karlsruhe, Germany

Résumé : Les conditions optimales de mesure de figures de pôles ont été recherchées en utilisant différentes variantes de montage (utilisation d'un monochromateur, détecteur en énergie dispersive, incidence réduite) et en analysant divers matériaux (alliage de cuivre, acier bas carbone, couches minces de TiN). La qualité des données mesurées dans chaque cas est estimée à partir des coefficients de compatibilité calculés.

Abstract : The best conditions for measuring pole figures have been sought by using several kinds of diffraction arrangement (use of a monochromator, energy dispersive detector, reduced incidence) and by analysing several materials (copper alloy, low carbon steel, TiN thin films). The quality of the measured data is estimated from the compatibility coefficients which are calculated in each case.

\section{INTRODUCTION}

La connaissance de la microstructure, au sens large du terme, (taille de grains, densité de dislocations, contraintes internes, texture cristallographique, etc...) est nécessaire pour comprendre et prédire le comportement ou les propriétés physiques ou mécaniques de matériaux polycristallins. Lorsque ces propriétés sont anisotropes, la texture cristallographique est souvent la grandeur déterminante.

La texture cristallographique d'un polycristal est caractérisée par la F.D.O. (Fonction de Densité des Orientations), notée $\mathrm{f}(\mathrm{g})$, définie comme la fraction volumique du matériau, $\mathrm{dV} / \mathrm{Vo}$, en orientation $\mathrm{g}$ à dg près :

$$
f(g) d g=d V / V o
$$

L'orientation $g$ d'un cristallite est la rotation ${ }^{1}$ qui amène un repère lié à l'échantillon $(A, B, C)$ parallèle à un repère lié au cristal (par exemple [100], [010], [001] dans le cas d'un cristal cubique).

\footnotetext{
${ }^{1}$ en fait, lorsque le cristal a une symétrie non triclinique, 1'orientation est un ensemble de rotations équivalentes par symétrie (par exemple 24 rotations dans le cas de la symétrie cubique).
} 
Le moyen expérimental le plus classique et le plus simple d'acquérir des données pour calculer la F.D.O. est le goniomètre de diffraction à $\mathbf{4}$ cercles qui permet de mesurer des figures de pôles. De telles mesures ont été proposées dès la fin des années 40 [1,2]. Le montage en réflexion est présenté à la Figure 1. Le faisceau de rayons $X$, sensiblement parallèle, arrive sur l'échantillon sous un angle d'incidence $\theta$. Le faisceau diffracté, limité par un système de fentes, traverse un filtre (élimination de la raie $\mathrm{K}_{\beta}$ ) et est détecté à l'angle $2 \theta$. Les fentes verticales permettent de choisir le domaine $\Delta 2 \theta$ du pic de Bragg; les fentes horizontales, distantes de $h$, limitent la partie de l'anneau de Debye qui entre dans le détecteur et définit ainsi, dans la figure de pôles, la 'largeur' $\Delta \chi$ du petit domaine mesuré.

$$
\Delta \chi=\mathbf{h} /(2 \mathrm{R} \sin \theta)
$$

où $\mathbf{R}$ est la distance échantillon-fentes.

L'échantillon est animé de 2 mouvements de rotation : déclinaison $\chi$ et azimuth $\phi$ qui permettent d'amener toute direction $y_{j}$ de l'échantillon parallèlle au vecteur de diffraction.

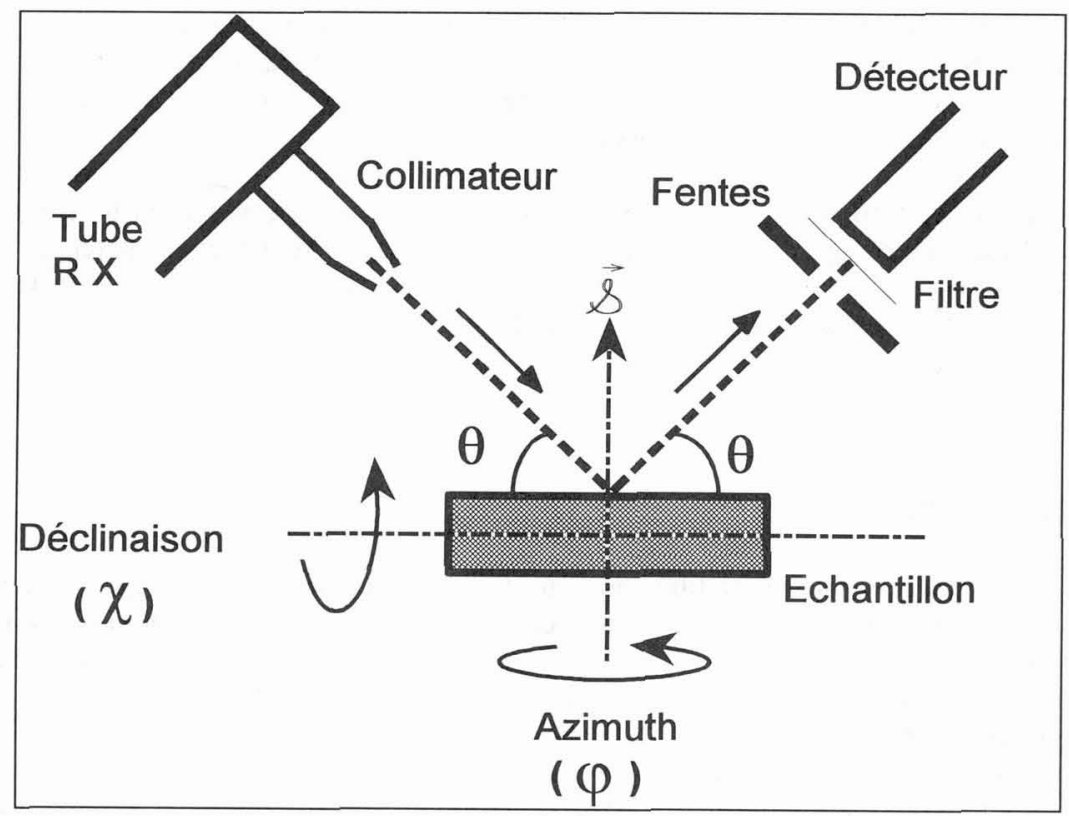

Figure 1 : Montage en réflexion pour la mesure d'une figure de pôles.

Une figure de pôles, $I_{h i}\left(y_{j}\right)$, est donc un ensemble de données qui fournissent lintensité diffractée par les plans dont la normale hi est parallèle à une direction yj repérée dans l'échantillon. Pour une répartition régulière des directions $y_{j}$, une figure de pôles est un ensemble de 1000 à 2000 mesures selon les pas en déclinaison et azimuth choisis. Ces données doivent être corrigées du bruit de fond, de la défocalisation (aux grands angles de déclinaison une partie seulement du faisceau diffracté pénétre dans le détecteur) et, lorsque l'échantillon est 
mince, de la variation de volume diffractant et d'absorption. En effectuant une normalisation, on obtient la densité de pôles expérimentale $P_{h_{i}}^{\exp }\left(y_{j}\right)$ :

$$
\mathbf{P}_{\mathrm{h}_{\mathrm{i}}}^{\exp }\left(\mathrm{y}_{\mathbf{j}}\right)=\mathrm{Ni} \mathrm{I}_{\mathrm{hi}}\left(\mathrm{y}_{\mathbf{j}}\right)
$$

où $\mathrm{Ni}$ est le coefficient de normalisation déterminé soit par référence à un échantillon isotrope, soit par une méthode mathématique appropriée $[3,4,5,6]$. Le calcul de la F.D.O., f(g), est basé sur la résolution de l'équation intégrale qui relie cette fonction aux densités de pôles :

$$
\mathbf{P}_{h_{i}}^{\exp }\left(y_{j}\right)=\int_{h_{i} / / y_{j}} f(g) d g
$$

Le souhait est bien sûr de déterminer la F.D.O. avec la meilleure précision possible, ce qui implique de mesurer et corriger les figures de pôles dans des conditions optimales. Si cette question apparaît de façon générale dans toutes les études de texture, elle prend un relief particulier dans le cas de 'matériaux nouveaux'. Par 'matériaux nouveaux' on entend par exemple les dépôts ou couches minces polycristallimes ou les multi-matériaux.

Dans cet article les résultats de différents tests, visant à définir les meilleures conditions de mesure pour les figures de pôles, sont présentés. Ces tests ont porté sur les trois points suivants :

- utilisation d'un monochromateur dans le montage de mesure de figures de pôles en réflexion

- utilisation de différents types de détecteurs

- utilisation d'un montage (assymétrique) en incidence réduite

Les matériaux utilisés lors de ces tests ont été d'une part des 'matériaux classiques' (alliage de cuivre et tôle d'acier à bas carbone) et d'autres part des 'matériaux nouveaux' (couches minces de TiN entre 20 et $600 \mathrm{~nm}$ d'épaisseur).

Pour comparer deux ensembles de mesures, il faut bien sûr disposer d'un critère permettant de discuter la qualité de ces données. Dans le domaine de l'analyse quantitative des textures, il s'agit des coefficients de compatibilité, souvent improprement appelés coefficients d'erreur. Lorsque la F.D.O., f(g), a été calculée à partir des densités de pôles expérimentales, il est possible de recalculer les densités de pôles, $P_{h_{i}}^{r e c}\left(y_{j}\right)$, pour tout $h_{i}$ et tout $y_{j}$. Si les données ne contiennent aucune erreur les densités de pôles recalculées sont identiques aux densités de pôles expérimentales pour les plans $h_{i}$ et les directions $y_{j}$ considérés. Dans le cas contraire, $\mathbf{f}(\mathrm{g})$ est une 'solution moyenne' à partir de données entachées d'erreur et les densités de pôles recalculées et expérimentales différent. C'est cette différence que quantifient les coefficients d'incompatibilité. Ils sont définis de la façon suivante :

$$
\operatorname{RPO}\left(h_{i}\right)=(1 / N 0) \Sigma\left(\left|P_{h_{i}}^{\exp }\left(y_{j}\right)-P_{h_{i}}^{r e c}\left(y_{j}\right)\right|\right) / P_{h_{i}}^{\exp }\left(y_{j}\right)
$$

où la somme porte sur l'ensemble No des valeurs telles que $P_{h_{i}}^{\exp }\left(y_{j}\right)>0$

$$
\operatorname{RP1}\left(h_{i}\right)=(1 / N 1) \Sigma\left(\left|P_{h_{i}}^{\exp }\left(y_{j}\right)-P_{h_{i}}^{r e c}\left(y_{j}\right)\right|\right) / P_{h_{i}}^{\exp }\left(y_{j}\right)
$$

où la somme porte sur l'ensemble N1 des valeurs telles que $P_{h_{i}}^{\exp }\left(y_{j}\right)>1$. 
Les coefficients $R P 0\left(h_{\mathfrak{j}}\right)$ sont particulièrement sensibles à la reproduction, dans les densités de pôles recalculées, des faibles valeurs alors que les coefficients $R P 1\left(h_{j}\right)$ sont particulièrement sensibles à la reproduction des pics. On notera respectivement $R P 0$ et RP1, la moyenne de ces coefficients pour un ensemble de figures de pôles.

Ce sont donc ces coefficients qui seront utilisés dans la suite pour discuter la qualité d'un ensemble de mesures de figures de pôles.

\section{TESTS SUR UN ALLIAGE DE CUIVRE}

Pour cet alliage, 4 figures de pôles ((111), (200), (220) et (311)) ont été mesurées en réflexion jusqu'à un angle de déclinaison $\chi=77.5^{\circ}$, pour le montage classique d'une part (cf. Figure 1) et pour un montage similaire où l'ensemble fentes-filtre est remplacé par un monochromateur arrière (monochromateur plan $\mathrm{LiF}$, monochromateur plan graphite, monochromateur courbe graphite). La F.D.O. ainsi que les coefficients de compatibilité ont été calculés à partir des données obtenues pour chacun des quatre montages. La Figure 2a) montre lune des figures de pôles, normalisée, mesurée (cas du montage avec monochromateur LiF plan) ainsi que la densité de pôles recalculée correspondante (Figure $2 b$ )). On reconnaît un maximum autour de l'orientation laiton (011)[211] et une symétrie d'échantillon orthorhombique induite par le laminage. La Figure 2c) montre cette même figure de pôles (200) expérimentale pour le montage conventionnel sans monochromateur. On constate que les pics à la périphérie sont très atténués dans ce dernier cas, ce qui correspond très vraisemblablement à une correction de défocalisation insuffisante.

Plus globalement, la table I donne les coefficients de compatibilité moyens pour l'ensemble des 4 figures de pôles dans chaque cas.

\begin{tabular}{|l|l|l|}
\hline \multicolumn{1}{|c|}{ Alliage $\mathrm{Cu}$} & RP0 & RP1 \\
\hline montage conventionnel & 32.8 & 14.1 \\
\hline montage avec mono $\lambda$ LiF plan & 26.4 & 15.1 \\
\hline montage avec mono $\lambda$ graphite plan & 40.9 & 14.9 \\
\hline montage avec mono $\lambda$ graphite courbe & 44.1 & 18.4 \\
\hline
\end{tabular}

Table I : coefficients de compatibilité moyens obtenus pour chaque type de montage

On constate que ces coefficients sont très voisins pour le montage conventionnel et le montage avec monochromateur plan $\mathrm{LiF}$ alors qu'ils se dégradent quelque peu pour les deux autres montages. Cette dégradation s'explique par le fait que pour les monochromateurs graphite la défocalisation apparait à des angles de déclimaison plus faibles (à cause de la taille du cristal) et devient très importante pour les grands angles de déclinaison; la correction de cet effet, à partir de mesures sur une poudre c.a.d. de données elles-même entachées d'erreur, ne permet pas 'd'effacer' complètement cet inconvénient. Les courbes de défocalisation utilisées pour la correction des données, mesurées pour une poudre de cuivre, sont présentées à la Figure 3.

Globalement on peut conchure que l'adjonction d'un monochromateur bien choisi n'apporte pas d'amélioration sensible dans le cas d'un tel matériau. 

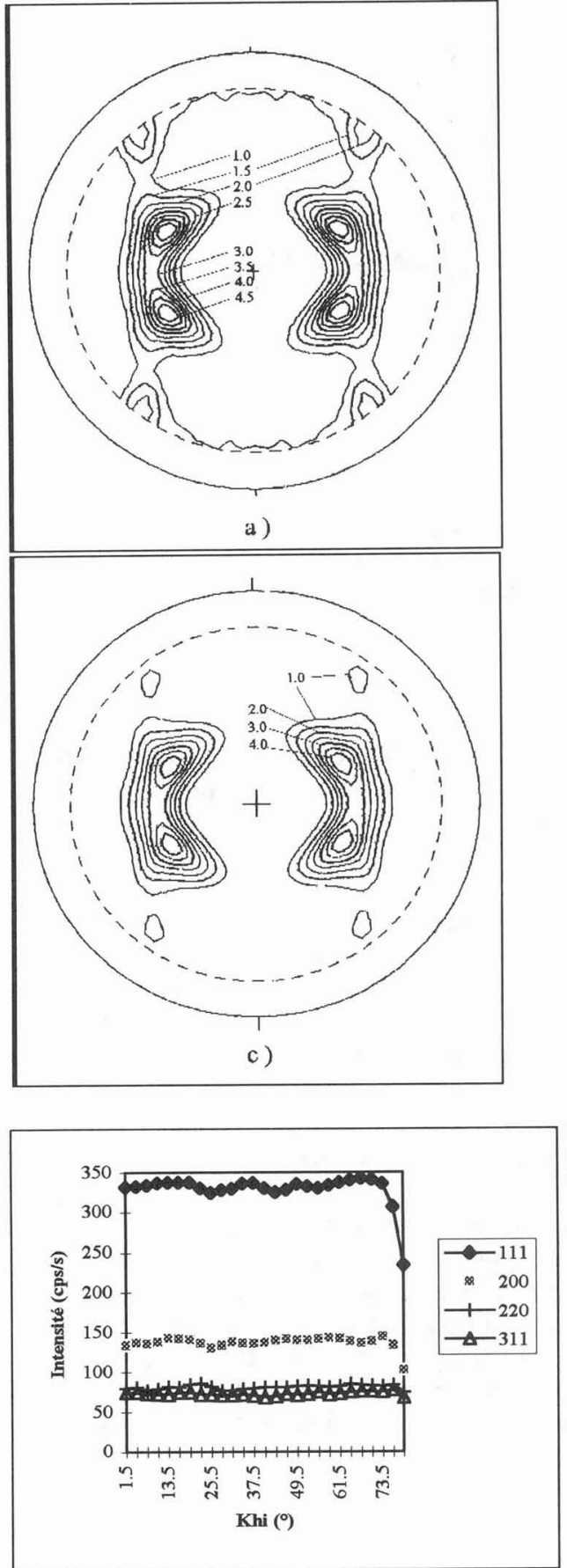

a)

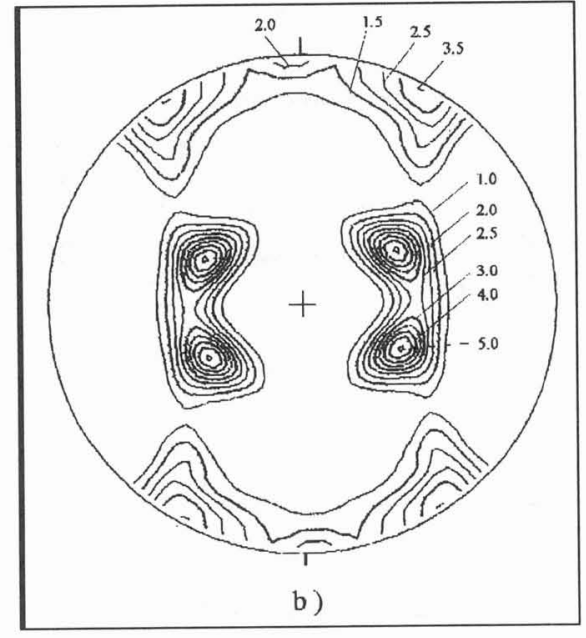

Figure 2: Figures de pôles de l'alliage de cuivre :

a ) (200) expérimentale mesurée avec monochromateur plan LiF.

b ) (200) recalculée

c ) (200) expérimentale mesurée avec montage conventionnel.

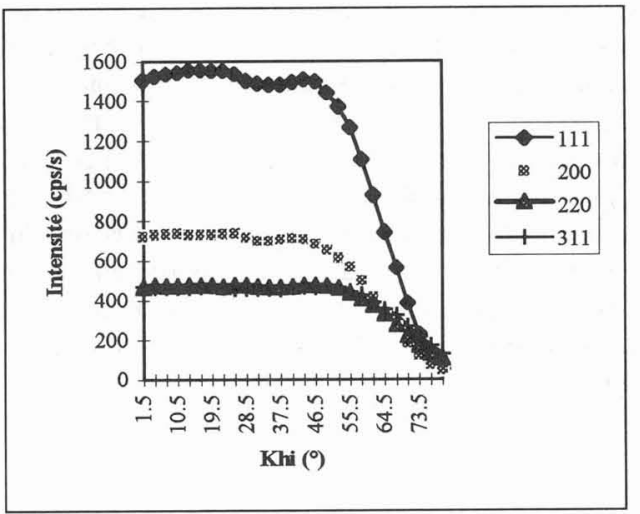

b) 


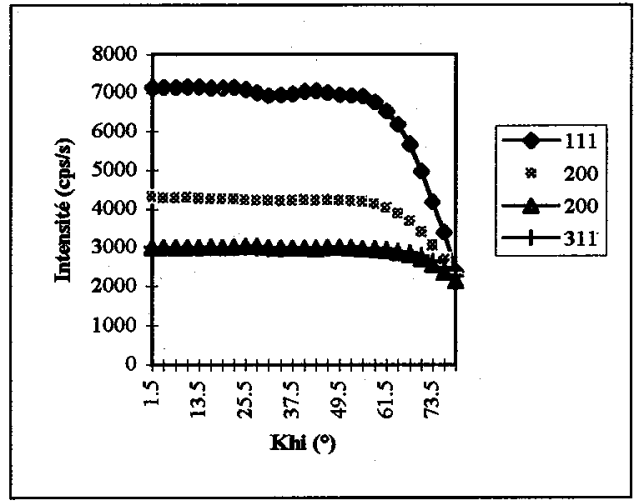

c)

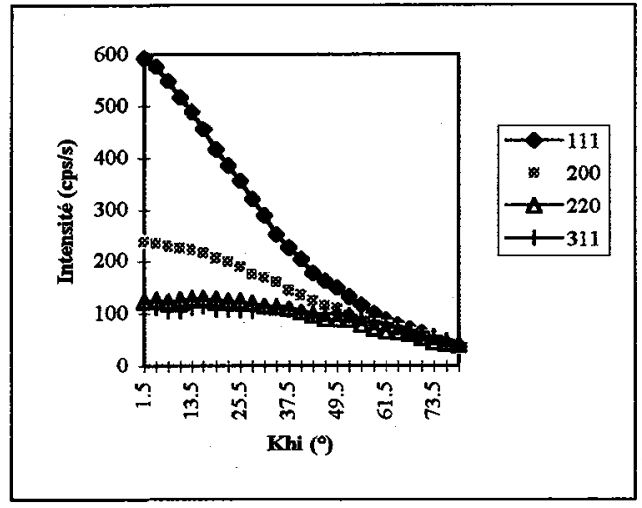

d)

Figure 3 : Courbes de défocalisation mesurées pour une poudre de cuivre pour montage :

a ) avec monochromateur plan LiF; b ) avec monochromateur plan de graphite.

c) conventionnel; d ) avec monochromateur courbe de graphite.

\section{TESTS SUR UN ACIER BAS CARBONE}

Dans le cas d'un acier à bas carbone laminé, trois figures de pôles en réflexion, (110), (200) et (211), ont été mesurées jusqu'à un angle de déclinaison maximum $\chi=77.5^{\circ}$ pour 5 montages différents. Les 5 montages utilisés sont les suivants :

- montage conventionnel avec radiation $\mathrm{Cu}$

- montage conventionnel avec radiation Co

- montage avec radiation $\mathrm{Cu}$ et monochromateur arrière plan LiF

- montage avec radiation $\mathrm{Cu}$ et monochromateur arrière plan graphite

- montage conventionnel avec radiation Cu et détecteur EDX (énergie dispersive)

On sait que la radiation du cuivre provoque une certaine fluorescence de l'échantillon d'acier et donc un bruit de fond important. Il s'agit donc d'examiner, pour un tel cas, si l'utilisation d'un monochromateur arrière ou d'un détecteur en énergie dispersive est à même d'éliminer cet inconvénient et de conduire à des données de bonne qualité. Les résultats sont comparés à ceux obtenus pour un montage conventionnel mais avec une radiation Co qui atténue très sensiblement ce phénomène de fluorescence.

L'une des figures de pôles mesurées (montage avec monochromateur plan LiF) ainsi que la densité de pôles correspondante recalculée sont présentées à la Figure 4.

Les résultats globaux, en termes de coefficients de compatibilité moyens, sont reportés à la table II. On constate que, pour la radiation $\mathrm{Cu}$, le montage avec monochromateur arrière plan LiF et surtout l'utilisation d'un détecteur en énergie dispersive améliore très sensiblement les résultats par rapport au cas du montage conventionnel. Toutefois lutilisation d'une radiation bien adaptée, ici celle du cobalt, dans le montage conventionnel foumit des résultats encore plus précis. 


\begin{tabular}{|l|c|c|}
\hline \multicolumn{1}{|c|}{ Acier bas carbone laminé } & RP0 & RP1 \\
\hline montage conventionnel tube $\mathrm{Cu}$ & 26.8 & 10.5 \\
\hline montage conventionnel tube $\mathrm{Co}$ & 8.9 & 4.2 \\
\hline montage conventionnel tube $\mathrm{Cu}$, détecteur EDX & 10.1 & 5.3 \\
\hline montage tube $\mathrm{Cu}$, mono $\lambda$ arrière plan $\mathrm{LiF}$ & 15.7 & 6.9 \\
\hline montage tube $\mathrm{Cu}$, mono $\lambda$ arrière plan graphite & 21.2 & 9.0 \\
\hline
\end{tabular}

Table II : coefficients de compatibilité moyens obtenus pour chaque type de montage
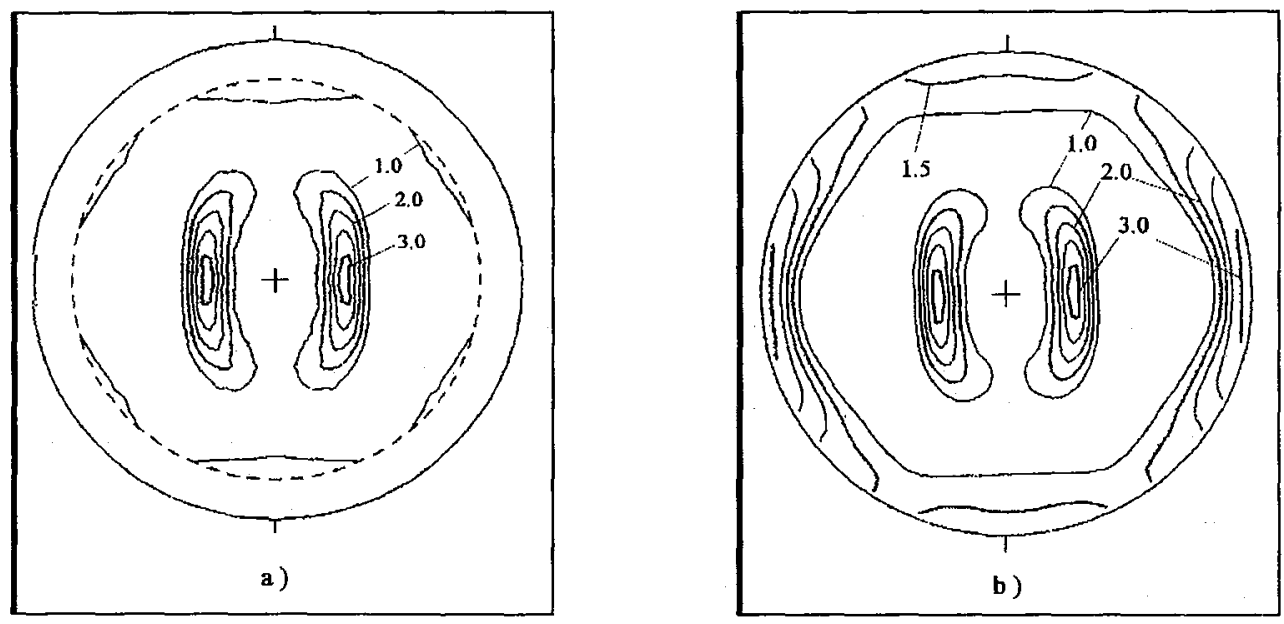

Figure 4 : Figures de pôles de l'échantillon d'acier :

a ) (110) expérimentale mesurée avec monochromateur plan LiF et b ) (200) recalculée.

\section{TESTS SUR DES COUCHES MINCES DE TIN}

Les échantillons sont ici des couches minces de TiN (épaisseurs 20,50,75,100,300 et $600 \mathrm{~nm}$ ) déposées par PVD sur un substrat monocristallin de silicium ${ }^{2}$. La difficulté provient dans ce cas du faible volume éclairé par les rayons $X$ donc du faible volume diffractant pour chaque point d'une figure de pôles. Il est possible d'augmenter ce volume diffractant en travaillant avec un montage asymétrique où le faisceau incident arrive sur l'échantillon sous un angle d'incidence réduit $\theta-\omega[7,8]$ comme le montre la Figure 5 . L'inconvénient de ce montage est que la figure de pôles ainsi mesurée présente une zone centrale 'aveugle' de rayon $\omega$. Dans un tel cas, il s'agit donc de trouver le meilleur compromis entre les deux effets antagonistes : amélioration de la statistique de comptage en incidence réduite et perte d'information dans la partie centrale des figures de pôles. Ce compromis dépend en partie de la texture de la couche mince étudiée.

\footnotetext{
${ }^{2}$ de telles couches sont utilisées dans des plaquettes électroniques comme barrièrres de diffusion entre le silicium et les conducteurs en aluminium.
} 


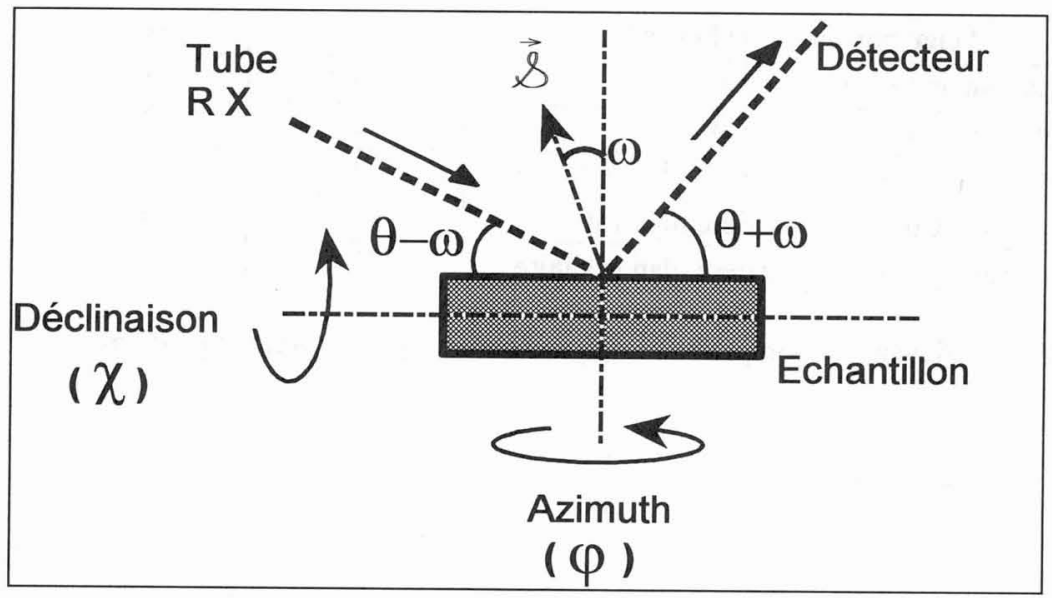

Figure 5 : montage en incidence reduite pour mesure de tigure de pöles en rètlexion

D'autre part, l'utilisation d'un monochromateur s'avère indispensable pour éviter des pics de diffraction parasites, dûs au substrat monocristallin, dans les figures de pôles de TiN.

La Figure 6 a) montre la figure de pôles (111) d'une couche mince de Tin d'épaisseur $600 \mathrm{~nm}$ pour un montage en incidence normale avec un monochromateur arrière plan $\mathrm{LiF}$. On reconnaît qu'il s'agit d'une texture de fibre avec les axes [111] orientés de façon préférentielle parallèlement à $\mathrm{DN}$, la direction normale à la surface de la couche. Les Figures $6 \mathrm{~b}$ ) et $6 \mathrm{c}$ ) montrent la figure de pôles expérimentale (200) mesurée respectivement avec et sans monochromateur arrière. On reconnait clairement, dans ce dernier cas, que le substrat monocristallin a donné lieu à 4 pics 'parasite' intenses et très fins; ils sont dus en fait à la diffraction de la raie $\mathrm{K}_{\beta}$ par les plans (220) du substrat.

Pour cet échantillon deépaisseur 600nm, 3 figures de pôles $((111),(200)$, et (220)) ont été mesurées pour différents montages (cf. Table III).

\begin{tabular}{|l|c|c|}
\hline \multicolumn{1}{|c|}{ TiN - 600nm } & RP0 & RP1 \\
\hline montage conventionnel (incidence $\theta$ ) & 80.7 & 27.4 \\
\hline montage mono $\lambda$ arrière LiF (incidence $\theta$ ) & 33.0 & 10.1 \\
\hline montage mono $\lambda$ graphite arrière (incidence $\theta$ ) & 89.6 & 11.3 \\
\hline montage mono $\lambda$ LiF avant (incidence $\theta$ ) & 32.1 & 2.8 \\
\hline
\end{tabular}

Table III : coefficients de compatibilité moyens obtenus pour chaque type de montage

II apparaît clairement que lutilisation d'un monochromateur est favorable, en particulier lorsqu'il est positionné à la sortie du tube de rayons $\mathrm{X}$.

Pour des échantillons de TiN de différentes épaisseurs, des mesures de figures de pôles ont été répétées en réduisant l'angle d'incidence $\left(\omega=0^{\circ}, 5^{\circ}, 10^{\circ}\right.$, et $15^{\circ}$; montage avec monochromateur avant) $[8,9]$. La Figure 7 rassemble les valeurs des coefficients de compatibilité déterminés dans chaque cas. La tendance est la suivante : aux faibles épaisseurs $(20,50 \mathrm{~nm})$, la réduction 
de lincidence, c.a.d. pour $\omega$ croissant, les coefficients de compatibilité décroissent; aux plus grandes épaisseurs au contraire, ces coefficients augmentent avec $\omega$. Pour ce type de texture, le montage en incidence normale est donc préférable pour des épaisseurs supérieures à environ $100 \mathrm{~nm}$, alors que pour des épaisseurs inférieures l'amélioration de la qualité des données obtenue en augmentant quelque peu le volume diffractant (incidence réduite) l'emporte sur l'inconvénient de perte d'information au centre des figures de pôles.
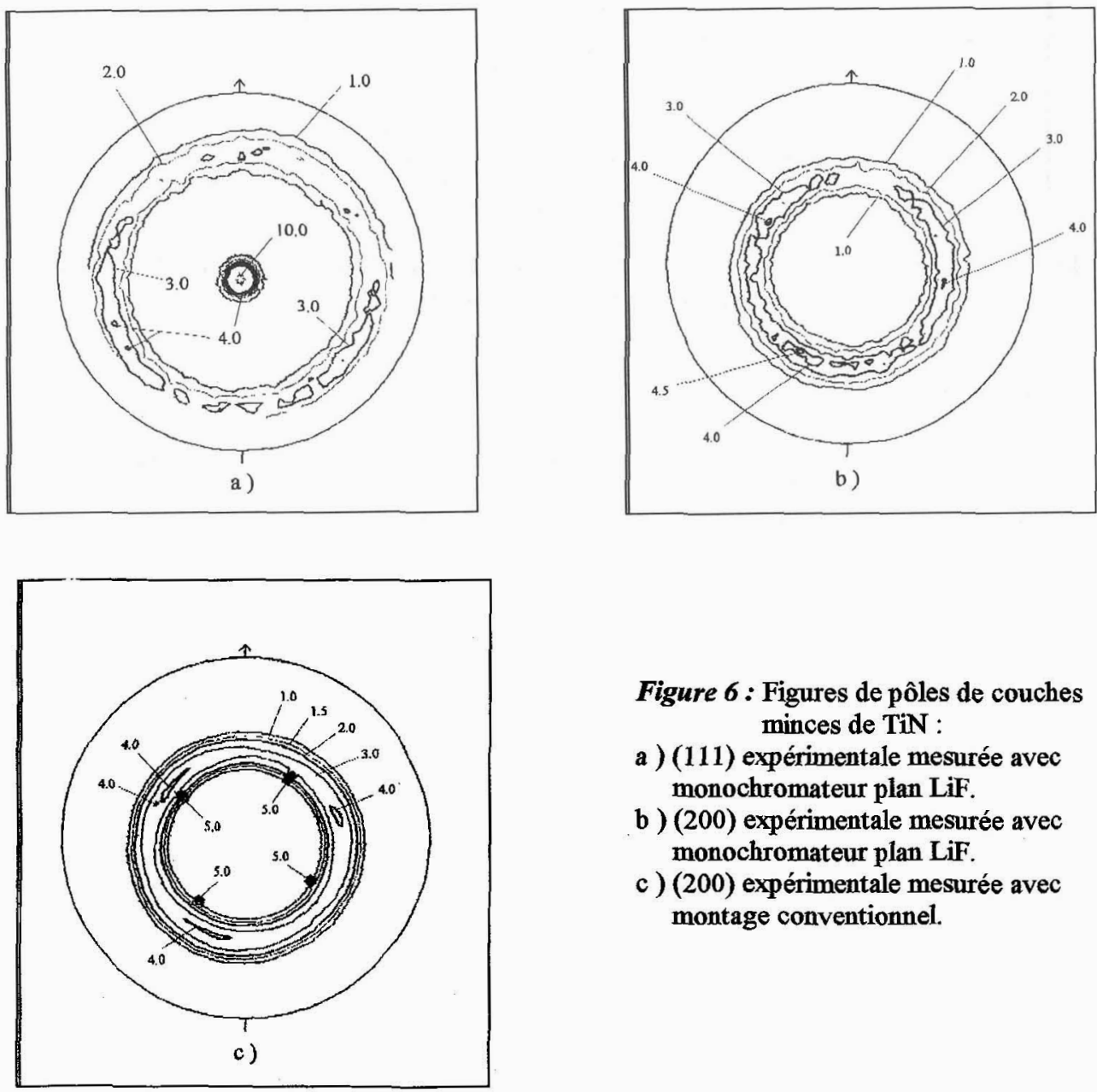

Figure 6: Figures de pôles de couches minces de TiN :

a ) (111) expérimentale mesurée avec monochromateur plan LiF.

b ) (200) expérimentale mesurée avec monochromateur plan LiF.

c) (200) expérimentale mesurée avec montage conventionnel. 


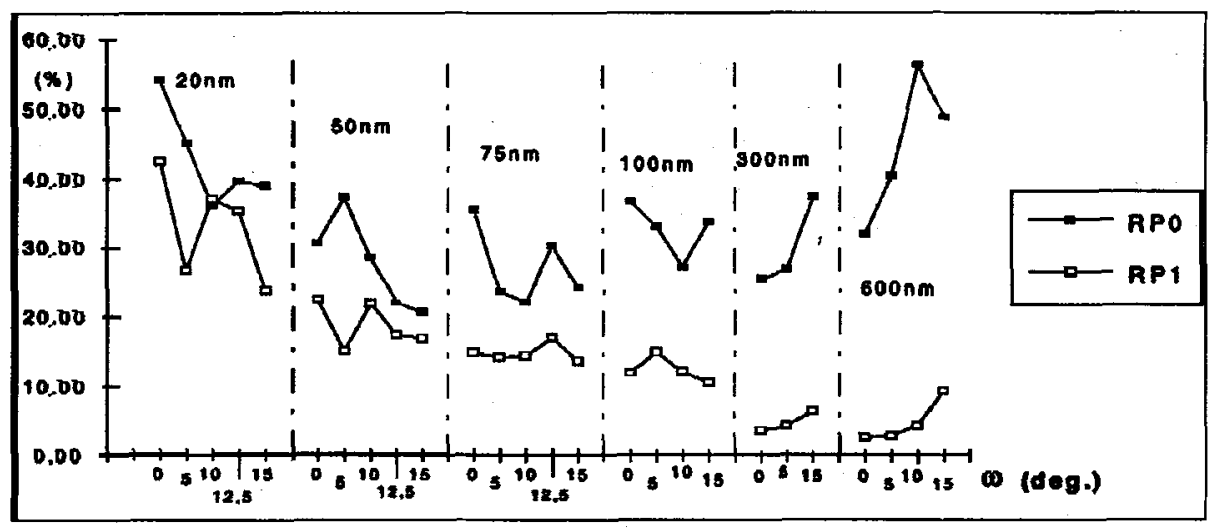

Figure 7 : coefficients de compatibilité RP0 et RP1 de couches minces TiN de différentes épaisseurs en fonction de l'angle $\omega$ de réduction d'incidence

\section{CONCLUSION}

Les tests effectués sur divers matériaux permettent de dégager quelques conclusions :

i) l'utilisation d'un monochromateur ne s'impose que pour des multi-matériaux (par exemple cas substrat-couche mince). Pour des matériaux 'classiques', le choix d'une radiation bien adaptée, c.a.d. qui évite une trop forte fluorescence, ainsi que l'utilisation d'un filtre devant le détecteur permettent d'obtenir des figures de pôles de qualité similaire à celle obtenue pour un montage avec monochromateur. Par contre un monochromateur arrière a un effet bénéfique si la fluorescence est importante.

ii) bien que ce point n'ait pas été examiné de façon extensive, il apparaît, dans le cas examiné ici, que l'utilisation d'un détecteur en énergie dispersive est favorable pour la mesure de figure de pôles. Ceci est lié au fait qu'un tel détecteur a très peu de bruit de fond; la correction correspondante est donc aisée alors que pour d'autres types de détecteur (scintillateurs, compteurs proportionnels) cette correction utilise des mesures parfois délicates à réaliser ou imprécises (mesures décalées du pic de Bragg à différentes déclinaisons par exemple). Une autre étude [10] semble montrer que l'utilisation d'un détecteur linéaire est favorable pour la qualité des figures de pôles, la raison essentielle étant encore une fois que la correction de bruit de fond pour chaque mesure peut être réalisée avec précision.

iii) dans le cas de couches minces, le montage en incidence réduite qui augmente le volume diffractant mais engendre une perte d'information au centre de la figure de pôles, est favorable aux faibles épaisseurs et défavorable aux plus grandes épaisseurs. Dans le cas de couches minces polycristallines de TiN avec des textures de fibre [111]// DN, cette 'frontière' se situe à une épaisseur d'environ $100 \mathrm{~nm}$.

\section{Références}

[1] Schulz L.G., J. Appl. Phys., 20, (1949), p. 1030.

[2] Decker B.F., Asp E.T. and Harker D., J. Appl. Phys., 19, (1948), p. 388

[3] Van Houtte P., Mater. Sci. Eng.,43, (1980), p.7 
[4] Liang Z., Xu J. et Wang F., Proceedings of ICOTOM 6, éd. The Iron and Steel Institute of Japan, (1981), p1259

[5] Dahms M. et Bunge H.J., Textures and Microstructures, 6, (1986), p. 167

[6] Wagner F. et Humbert M., Textures and Microstructures, 7, (1987), p. 115

[7] Heizmann J.J., Vadon A., Schlatter M. et Bessieres J., Adv. in X-ray Anal., 32, (1989), p. 285

[8] Moreau B., Wagner F. et Göbel H, Proceedings of ICOTOM 10, éd. Trans Tech Publication, (1994), p. 159

[9] Moreau B., Thèse de Doctorat d'Université, Université de Metz, (1993)

[10] Wcislak L., Bunge H.J. et Nauer-Gerhardt, Zeit. Metallkd., 84, (1993), p. 479 